\title{
BMJ Open Protocol for a scoping review of existing policies on the prevention and control of obesity across countries in Africa
}

\author{
Theodosia Adom, ${ }^{1,2}$ Thandi Puoane, ${ }^{2}$ Anniza De Villiers, ${ }^{3}$ André Pascal Kengne ${ }^{3,4}$
}

To cite: Adom T, Puoane T, De Villiers A, et al. Protocol for a scoping review of existing policies on the prevention and control of obesity across countries in Africa. BMJ Open 2017;7: e013541. doi:10.1136/ bmjopen-2016-013541

- Prepublication history and additional material is available. To view please visit the journal (http://dx.doi.org/ 10.1136/bmjopen-2016013541).

Received 19 July 2016 Revised 11 November 2016 Accepted 20 December 2016

\section{CrossMark}

\footnotetext{
${ }^{1}$ Nutrition Research Centre, Radiological and Medical Sciences Research Institute, Ghana Atomic Energy Commission, Ghana

${ }^{2}$ Faculty of Community and Health Sciences, School of Public Health, University of the Western Cape, Bellville, South Africa

${ }^{3}$ Non-communicable Disease Research Unit, South African Medical Research Council, South Africa

${ }^{4}$ University of Cape Town, South Africa
}

Correspondence to Theodosia Adom; theo.adom@gmail.com

\section{ABSTRACT}

Introduction: The obesity epidemic is a public health challenge for all, including low-income countries. The behavioural patterns known to contribute to the rise in obesity prevalence occur in an environmental context which is not conducive for healthy choices. A policy approach to obesity prevention constitutes a form of public intervention in that it extends beyond individuals to influence entire populations and is a mechanism for creating healthier environments. Little is known about obesity prevention policies in Africa. This scoping review seeks to examine the nature, extent and range of policies covering obesity prevention in Africa in order to assess how they align with international efforts in creating less obesogenic environments. This will help identify gaps in the approaches that are adopted in Africa.

Methods and analysis: Using the Arksey and O'Malley's scoping methodological framework as a guide, a comprehensive search of MEDLINE (PubMed), MEDLINE (EbscoHost) CINAHL (EbscoHost), Academic Search Complete (EbscoHost) and ISI Web of Science (Science Citation Index) databases will be carried out for peer reviewed journal articles related to obesity prevention policies using the African search filter. A grey literature search for policy documents and reports will also be conducted. There will be no language and date restrictions. Eligible policy documents and reports will be obtained and screened using the inclusion criteria. Data will be extracted and results analysed using descriptive numerical summary analysis and qualitative thematic analysis.

Ethics and dissemination: No primary data will be collected since all data that will be presented in this review are based on published articles and publicly available documents, and therefore ethics committee approval is not a requirement. The findings of this systematic review will be presented at workshops and conferences; and will be submitted for publication in peer-reviewed journal. This will also form a chapter of a PhD thesis.

\section{INTRODUCTION}

Obesity/overweight is a major modifiable risk factor for non-communicable diseases (NCDs) including coronary heart disease,

\section{Strengths and limitations of this study}

- This review approach will provide a broad overview of obesity prevention policies and describe key concepts and issues across Africa.

- The review will provide information on the role of these policies in creating less obesogenic environments to further inform future policy directions.

- Some of these policies may not be available as published documents but all efforts will be made to obtain them through key regional and national representatives.

type II diabetes and many cancers. ${ }^{1}$ According to the World Health Organization (WHO) nearly $80 \%$ of NCD deaths occur in low-income and middle-income countries except Africa. Current projections however indicate that by 2020 the largest increases in NCD-related deaths will occur in Africa and by 2030 , these deaths are projected to exceed the combined deaths from communicable and nutritional diseases, and maternal and perinatal deaths. ${ }^{2}$

Although there are many causes, the behavioural patterns known to contribute significantly to the rise in obesity prevalence include increased consumption of energydense foods, low consumption of fruits and vegetables, increased sedentary lifestyle and low level of physical activity. ${ }^{3}$ These behaviours occur in an environmental context which does not support healthy choices also known as 'obesogenic' environment, such as promotion of high density of fast food outlets and restaurants, promoting cheap but energy dense foods and poor urban planning which does not support active transport. ${ }^{4}$

There is the need to create healthy environments as a way to manage the epidemic; policies and regulations are needed to drive the environment and social changes that would have a sustainable impact on controlling obesity. A policy approach to obesity 
prevention constitutes a form of public intervention in that it extends beyond individuals' effects to influence entire populations and is a mechanism for creating healthier environments. ${ }^{5}$

In response to the global burden of NCDs, the WHO in 2004 adopted the Global Strategy on Diet, Physical Activity and Health (DPAS) ${ }^{1}$ with an overall goal to 'promote and protect health by guiding the development of an enabling environment for sustainable actions at individual, community, national and global levels, that when taken together, will lead to reduced disease and death rates related to unhealthy diet and physical inactivity'. The DPAS calls on Member States to develop and implement national policies and programmes depending on their national circumstances to improve diets and promote physical activity, two major risk factors of obesity. The DPAS, together with Global Strategy for the Prevention and Control of Non-communicable Diseases ${ }^{6}$ and 2008-2013 Action Plan for the Global Strategy for the Prevention and Control of Non-communicable Diseases $^{7}$ are some of the international efforts to control and prevent NCDs.

The role of national governments is seen in providing leadership as a sign of commitment; developing, implementing and monitoring a set of policy instruments that make environments less obesogenic and more health promoting; and securing increased and continuing funding to create healthy environments and encourage physical activity and healthy eating. ${ }^{8}$

There is considerable evidence in support of policy in obesity intervention and this is based largely on studies from high-income countries such as the US, Australia and Europe. ${ }^{9-11}$ Some of these policy instruments are laws, regulations, taxes, subsidies and social marketing campaigns that are population-based affecting both children and adults and may target different settings such as schools, health institutions and workplaces. Low-income and middle-income countries may have distinct cultures and infrastructure that limit generalisation of strategies from high-income countries. Moreover, low-income countries may lack the financial resources to implement policies that have shown considerable results in highincome countries. There is also limited information on obesity prevention policies in Africa.

\section{OBJECTIVE}

This scoping review seeks to examine the nature, extent and range of policies covering obesity prevention in Africa in order to assess how they align with international efforts in creating less obesogenic environments. This will help identify gaps in the approaches that are adopted in Africa.

\section{METHODS}

The methods for this review will follow a scoping review methodological framework. ${ }^{12}$ This describes a 6-stage approach to the review process, the sixth stage being optional. Unlike systematic reviews which answer specific research questions by collating all empirical evidence with predefined eligibility criteria, scoping reviews tend to cover broader topics in the research area of interest to map relevant literature, key concepts and identify research gaps. ${ }^{12}{ }^{13}$ Scoping reviews have unclear boundaries at the outset; prespecified eligibility criteria are therefore provisional and may be refined and applied iteratively during the review with emerging knowledge of the existing literature.

\section{Stage 1: Identifying the research questions}

Based on the literature and the WHO documents, ${ }^{16}$ key research questions were derived (box 1). The Preferred Reporting Items for Systematic Reviews and MetaAnalyses (PRISMA)-Equity 2012 Extension ${ }^{14}$ will be used to identify equity-focused questions and operational definitions to be addressed in this review.

\section{Inclusion criteria}

1. National policy documents, reports and the literature produced or published that aim at reducing the risk factors of NCDs such as unhealthy diets, physical inactivity, overweight and obesity or that form part of larger chronic diseases prevention strategies, or to prevent and control obesity.

2. No language and date restrictions are set.

\section{Exclusion criteria}

Policies that are being implemented in other countries outside Africa

\section{Box 1 Research questions and operational definitions}

Research question

1. Which obesity prevention policies are being addressed in the documents?

- Individual-targeted (consumer information and labelling)

- Physical environments

- Economic environments

- Political environments

- Sociocultural environments

- Promotions or educational campaigns

2. Who are the target populations being addressed?

- Individuals (children, adolescents, adults)

- Family

- Subpopulations (age groups)

- Settings (home, schools, health institutions, workplaces)

- Society (industry, general public)

3. How is equity addressed in the obesity prevention literature?

- Equity is explicitly stated in the objective or in the analysis

- Population characteristics such as gender and socioeconomic status

4. What are the barriers and/or facilitators to the implementation of these policies?

Barriers and facilitators as identified by authors

5. Is there evidence of effectiveness of interventions?

Effectiveness as identified by authors 


\section{Stage 2: Identifying relevant studies}

Two researchers will independently conduct a comprehensive and broad search of the following electronic databases of peer reviewed journal articles and online search registers related to obesity prevention policies using the African search filter: ${ }^{15}$ MEDLINE (PubMed), MEDLINE (EbscoHost) CINAHL (EbscoHost), Academic Search Complete (EbscoHost) and ISI Web of Science (Science Citation Index). Search terms will include keywords developed from Medical Subject Headings (MeSH) and 'nutrition', 'food', 'physical activity' in combination with 'policy', guideline', 'action plan', 'strategy,' 'regulation', 'law', relating to 'overweight', 'obesity', 'noncommunicable diseases'. Manual-searching of reference lists of relevant documents, and systematic reviews and meta-analyses will be carried out to identify additional studies that will not be captured by the electronic database search. A grey literature search for unpublished policy documents and reports will be undertaken from Google Scholar, relevant websites such as WHO, UNICEF, Ministries of Health and Departments of Health in different countries, OPENGREY, African Index Medicus (AIM), AFROLIB (WHO Regional Office Database for Africa), workshops and conference proceedings. Key persons at the health and education ministries of the countries will be contacted (at the WHO NCD offices) about existing policy documents and reports and copies obtained where these are available. A search strategy for PubMed database is attached (see online supplementary appendix 1). References will be exported and duplicates removed using citation management software.

\section{Stage 3: Study selection}

Two-step approach will be used to select the relevant literature. Two independent researchers will screen the titles, abstracts and executive summaries. Potentially relevant literature will be obtained and inclusion and exclusion criteria applied to check the eligibility. Documents that meet the criteria will be assessed for inclusion in the review. Any disagreement about the eligibility will be resolved by a third reviewer. Regular discussions will be held by team members to assess the progress of the process. ${ }^{13}$

\section{Stage 4: Charting the data}

Standardised data charting forms will be developed and used to extract data from included documents. Data will be charted and sorted according to these key issues and themes. Two researchers will independently extract the data and it will be compared. The data extracted will include: geographical location, country, author, year of publication, development of document (multisectoral and/private sector approach), type of document (national health policy, action plan, strategic plan and policy document), target populations (individuals, family and subpopulations), target settings (schools, healthcare institutions and industry), barriers/facilitators to implementation of policies and evidence of intervention effectiveness, if available. The listed data may be modified as reviewers become more familiar with the literature.

Stage 5: Collating, summarising and reporting the results To guide the assessment of the role of these policies in creating less obesogenic environments, the Analysis Grid for Environments Linked to Obesity (ANGELO) Framework $^{16 \quad 17}$ will be used to categorise policies into one of four environments: physical (what is available), economic (what are the costs), political (what are the rules) and sociocultural (what are the attitudes and beliefs); and two settings (macrosetting and microsetting). The ANGELO Framework is a common framework for understanding the obesogenicity of the environment. Data will be analysed and presented using descriptive numerical summary analysis and qualitative thematic analysis.

\section{CONCLUSION}

This scoping review will provide a broad overview of obesity prevention policies and describe key concepts and issues across Africa to further inform future policy direction. The results will help identify gaps in the approaches that are adopted in Africa, provide better understanding of the progress made and provide needed support for implementation, and the effectiveness of these policy interventions by governments across the continent.

Contributors APK and TA conceived the review approach. APK provided general guidance to the drafting of the protocol. TA drafted the manuscript. APK, TA, ADV and TP reviewed and revised the manuscripts. All authors have read and approved the final version of the manuscript.

\section{Competing interests None declared}

Provenance and peer review Not commissioned; externally peer reviewed.

Open Access This is an Open Access article distributed in accordance with the Creative Commons Attribution Non Commercial (CC BY-NC 4.0) license, which permits others to distribute, remix, adapt, build upon this work noncommercially, and license their derivative works on different terms, provided the original work is properly cited and the use is non-commercial. See: http:// creativecommons.org/licenses/by-nc/4.0/

\section{REFERENCES}

1. World Health Organization. Global strategy on diet, physical activity and health. Fifty-seventh World Health Assembly, Geneva, 17-22 May 2004. Resolutions and decisions, annexes. Geneva, World Health Organization, 2004.

2. World Health Organization. The global burden of disease: 2004 update. Geneva, World Health Organization, 2008.

3. Allender S, Cavill N, Parker M, et al. "Tell us something new!"- the response of planning and transport professionals to public health guidance on the built environment and physical activity. $J$ Public Health Policy 2009;30:102-16.

4. Swinburn B, Egger G. Preventive strategies against weight gain and obesity. Obes Rev 2002;3:289-301.

5. Brownson RC, Haire-Joshu D, Luke DA. Shaping the context of health: a review of environmental and policy approaches in the prevention of chronic diseases. Annu Rev Public Health 2006;27:341-70.

6. World Health Organization. 2008-2013 Action plan for the global strategy for the prevention and control of noncommunicable 
diseases. Geneva: World Health Organization, 2008. http://www. who.int/nmh/publications/9789241597418/en/ (accessed 7 Jul 2016).

7. World Health Organisation. Political Declaration of the High-level Meeting of the General Assembly on the Prevention and Control of Noncommunicable Diseases. 66th Session of the General Assembly, agenda item 117. A/66/L. 1 2011. http://www.who.int/nmh/events/un_ ncd summit2011/political declaration en.pdf

8. Lawrence $M$, Swimburn $\bar{B}$. The role of policy in preventing childhood obesity. In: Preventing childhood obesity: evidence policy and practice. Waters E, Swinburn BA, Seidell JC, Uauy R. Blackwell Publishing, 2010:203-11.

9. Chriqui JF. Obesity prevention policies in U.S. States and localities: lessons from the field. Curr Obes Rep 2013;2: $200-10$

10. Novak NL, Brownell KD. Role of policy and government in the obesity epidemic. Circulation 2012;126:2345-52.

11. Boehmer TK, Brownson RC, Haire-Joshu D, et al. Patterns of childhood obesity prevention legislation in the United States. Prev Chronic Dis 2007;4:A56.
12. Arksey H, O'Malley L. Scoping studies: towards a methodological framework. Int J Soc Res 2005;8:19-32.

13. Daudt HM, van Mossel C, Scott SJ. Enhancing the scoping study methodology: a large, inter-professional team's experience with Arksey and O'Malley's framework. BMC Med Res Methodol 2013;13:48.

14. Welch V, Petticrew M, Tugwell $P$, et al. The PRISMA-Equity Bellagio group. PRISMA-Equity 2012 extension: reporting guidelines for systematic reviews with a focus on health equity. PLoS Med 2012;9 (10):e1001333. http://dx.doi.org/10.1371/journal.pmed.1001333

15. Siegfried N, Clarke M, Volmink J. Randomized controlled trials in Africa of HIV and AIDS: descriptive study and spatial distribution. BMJ 2005;331:742-6.

16. Swinburn B, Egger G, Raza F. Dissecting obesogenic environments: the development and application of a framework for identifying and prioritizing environmental interventions for obesity. Prev Med 1999;29:563-70.

17. Mooney JD, Jepson R, Frank J, et al. Obesity prevention in Scotland: a policy analysis using the ANGELO framework. Obes Facts 2015;8:273-81. 